\title{
Mixed-valence behavior and strong correlation effects of metal phthalocyanines adsorbed on metals
}

\author{
S. Stepanow, ${ }^{1}$ P. S. Miedema, ${ }^{2}$ A. Mugarza,${ }^{1}$ G. Ceballos,${ }^{1}$ P. Moras,${ }^{3}$ J. C. Cezar, ${ }^{4}$ C. Carbone, ${ }^{3}$ \\ F. M. F. de Groot, ${ }^{2}$ and P. Gambardella ${ }^{1,5}$ \\ ${ }^{1}$ Catalan Institute of Nanotechnology (ICN-CIN2), UAB Campus, E-08193 Barcelona, Spain \\ ${ }^{2}$ Debye Institute of Nanomaterials Science, Utrecht University, NL-3584 CA Utrecht, The Netherlands \\ ${ }^{3}$ Istituto di Struttura della Materia, Consiglio Nazionale delle Ricerche, Area Science Park, I-34012 Trieste, Italy \\ ${ }^{4}$ European Synchrotron Radiation Facility, BP 220, F-38043 Grenoble, France \\ ${ }^{5}$ ICREA and Universitat Autònoma de Barcelona, E-08193 Barcelona, Spain
}

(Received 21 March 2011; published 9 June 2011)

\begin{abstract}
We investigate the hierarchy of local correlation and hybridization effects in metal-organic molecules adsorbed on metals. Using x-ray magnetic circular dichroism and ligand field multiplet calculations, we demonstrate that the $3 d$ electronic ground state of monolayer metal-phthalocyanine ( $\mathrm{CoPc}, \mathrm{FePc}$ ) on $\mathrm{Au}(111)$ is given by the coherent superposition of two charge states, $d^{n} E+d^{n+1}$, where $E$ represents a substrate electron antiferromagnetically coupled to the central metal ion and $d^{n}$ the many-body ionic orbital configuration of the unperturbed molecule. These results differ from previous models of hybrid metal-organic systems and provide a consistent description of their magnetic moments and Kondo physics in terms of spin and orbital multiplicity.
\end{abstract}

DOI: 10.1103/PhysRevB.83.220401

PACS number(s): 75.70.Ak, 73.20.Hb, 78.70.Dm, 81.07.Pr

The magnetic properties of transition-metal (TM) compounds depend critically on the competition between $d$-d electron correlation and covalency, that is, the transfer of charge between $d$-orbitals and delocalized ligand states. ${ }^{1}$ Charge transfer (CT) affects the magnetization of TM ions, their coupling through indirect exchange paths, as well as the conductivity of important classes of materials, including TM oxides, high- $T_{c}$ superconductors, and molecular complexes. Recently, CT processes that take place at the interface between magnetic molecules and metal surfaces have come under intensive attention. ${ }^{2-4}$ Transport measurements of single molecules trapped between metallic electrodes ${ }^{5}$ and layered metal/organic heterostructures ${ }^{6}$ have revealed the role played by interfacial hybridization in determining efficient charge and spin injection across TM complexes, a prerequisite to developing molecular spintronic devices. ${ }^{6,7}$ A comprehensive description of the electronic and magnetic properties of metal-organic hybrids, however, is complicated by the fact that CT can occur between TM and ligand orbitals, but also between any of these states and the electron reservoir of a supporting metal surface or electrode. Clearly, understanding such processes would be of great importance in modeling and controlling the properties of magnetic molecules interfaced with metals.

Density functional theory (DFT) provides the basis of our current understanding of hybrid metal-organic systems. ${ }^{2-4,8,9}$ Yet, due to the difficulty of modeling exchange and electron correlation phenomena using effective one-electron potentials, DFT affords only a partial description of the magnetic properties of TM complexes. ${ }^{1}$ Methods beyond standard DFT that include Coulomb repulsion through semiempirical Hubbard U terms have been successfully employed to describe the ground state of isolated molecules. ${ }^{10}$ However, DFT $+\mathrm{U}$ remains a mean-field scheme, which precludes a proper modeling of valence and spin fluctuations induced by CT.

A notable case where CT has a dramatic influence on the magnetism of a TM complex is that of Co-phthalocyanine (CoPc), a well-known molecule with spin $S=1 / 2$ (Ref. 11).
DFT predicts that CoPc adsorbed on $\mathrm{Au}(111)$ (Refs. 12 and 13), $\mathrm{Cu}(111)$ (Ref. 14), and even ferromagnetic Fe(110) films $^{2}$ assume a nonmagnetic $S=0$ configuration due to complete filling of the previously unpaired $d_{z^{2}}$ state owing to CT from the substrate. Experimental evidence supporting this interpretation, however, is rather indirect and based uniquely on the absence of Kondo resonances detected by scanning tunneling microscopy (STM). ${ }^{12,14}$ Moreover, spin-polarized STM of $\mathrm{CoPc} / \mathrm{Fe}(110)$ shows that spin-split molecule-surface hybrid states exist at the Co site even though the total magnetic moment calculated by DFT is zero. ${ }^{2}$

In light of these considerations, we have investigated the electronic structure of CoPc and FePc layers on $\mathrm{Au}(111)$ using $\mathrm{x}$-ray absorption spectroscopy (XAS) and x-ray magnetic circular dichroism (XMCD) combined with atomic multiplet calculations including ligand field and CT. This approach is complementary to DFT and STM, with the advantage of providing an appropriate description of electron correlation and a direct measurement of local TM magnetic moments. ${ }^{15}$ XMCD reveals that valence fluctuations entirely quench the $\mathrm{Co}$ moment upon deposition of $\mathrm{CoPc}$ on $\mathrm{Au}$, whereas the magnetic moment of FePc survives adsorption. We show that the ground state of $\mathrm{CoPc}$ and $\mathrm{FePc}$ in contact with a metallic substrate is best described by the coherent superposition of two many-electron states representing charge fluctuating from the substrate to the TM ion, with $L S$ multiplicity determined by electron correlation and ligand field effects of similar magnitude compared to the free molecule. Our results provide a consistent description of the magnetic properties of hybrid metal-organic complexes which differs from one-electron $a b$ initio methods and also from CT typically encountered in bulk TM coordination compounds (Fig. 1).

XAS and XMCD measurements were performed at beamline ID08 of the European Synchrotron Radiation Facility using linearly and circularly polarized light tuned to the $L_{2,3}$ absorption edges of $\mathrm{Co}$ and $\mathrm{Fe}$. Linearly polarized spectra were recorded at $300 \mathrm{~K}$ in order to reduce background features due to the extended x-ray absorption fine structure of Au. Circularly 
(a)

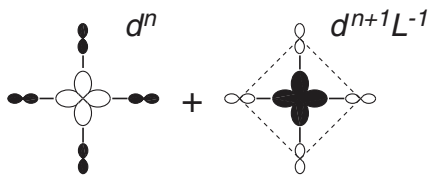

(b)

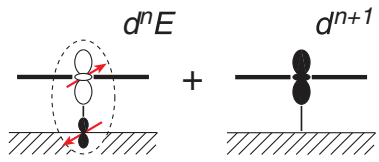

FIG. 1. (Color online) (a) Schematic of ligand-to-metal charge transfer in TM compounds. (b) Metal surface-to-CoPc charge transfer.

polarized $\mathrm{x}$-ray absorption spectra were measured at $8 \mathrm{~K}$ in total electron yield in a magnetic field of $5 \mathrm{~T}$ applied parallel to the $\mathrm{x}$-ray beam, both making an angle $\theta$ relative to the surface normal. The XMCD signal, defined as the difference between negative $\left(I^{-}\right)$and positive $\left(I^{+}\right)$circularly polarized absorption, is proportional to the expectation value of the TM magnetic moment projected onto the $\mathrm{x}$-ray incidence direction. $\mathrm{CoPc}$ and $\mathrm{FePc}$ films were evaporated in situ onto a sputter-annealed $\mathrm{Au}(111)$ single crystal held at room temperature in ultrahigh vacuum. STM and low-energy electron diffraction were used to monitor the planar growth of the Pc films prior to transfer into the XMCD chamber. ${ }^{16}$ To interpret the electronic and magnetic configuration of the TM ions, we employed CT multiplet theory ${ }^{15}$ implemented using a numerical code free of symmetry restrictions. ${ }^{17}$

Figure 2 shows the XAS and XMCD spectra of a fourlayer-thick film of CoPc (a), which we use as a reference to investigate CT effects in monolayer CoPc/Au(111) (b, c). In either case the molecules are oriented with their symmetry axis perpendicular to the surface plane, as evidenced by linear dichroism at the $N$ edge. ${ }^{16}$ The multilayer spectra are in agreement with XAS data reported earlier for thicker films. ${ }^{18,19}$ The XAS exhibits a strong natural linear dichroism, correlated with the low-symmetry environment of the Co ion, whereas the finite XMCD signal indicates the presence of a net $3 d$ magnetic moment localized on Co. The $3 d$ ground state of $\mathrm{CoPc}$ is approximately known. ${ }^{11}$ However, uncertainties still exist regarding the role of excited states mixed into the ground state. ${ }^{11,18,20}$ Here, taking advantage of XAS and XMCD simulations at low temperature, we are able to provide a description of the molecule electronic structure that settles previous ambiguities. Simulated spectra from ligand field multiplet calculations are shown in Fig. 2(a). Both the experimental XAS and XMCD are reproduced with excellent accuracy assuming a $d^{7}$ configuration of the Co ion and $D_{4 h}$ crystal-field symmetry with parameters $10 D_{q}=2.3$, $D_{s}=0.55$, and $D_{t}=0.20 \mathrm{eV}$, reducing the Slater-Condon integrals to $75 \%$ of their atomic value. The experimental $\mathrm{XMCD}$ intensity is much smaller compared to the simulations due to antiferromagnetic coupling between stacked $\mathrm{CoPc}$ molecules in multilayer films, ${ }^{21}$ but the spectral features are well reproduced. The ground-state symmetry is ${ }^{2} A_{1 g}$, that is, a low-spin configuration with $S=1 / 2$ and the unpaired electron located in the $a_{1 g}\left(d_{z^{2}}\right)$ orbital. The first excited state $\left({ }^{2} E_{g}\right)$ lies about $80 \mathrm{meV}$ above the ground state. Spin-orbit coupling mixes the ${ }^{2} A_{1 g}$ and ${ }^{2} E_{g}$ states, partly depopulating the $e_{g}$ orbital (23\% hole contribution) and filling the empty $a_{1 g}$ (20\%), thus resulting in a $\left(e_{g}\right)^{3.8}\left(b_{2 g}\right)^{2}\left(a_{1 g}\right)^{1.2}$ configuration. The depopulation of the $e_{g}$ states creates a finite in-plane orbital moment in the ground state, which is reflected by the larger amplitude of the integrated XMCD at grazing incidence $\left(\theta=70^{\circ}\right)$ compared to normal incidence $\left(\theta=0^{\circ}\right)$.

The XAS spectral shape changes dramatically for $\mathrm{CoPc}$ adsorbed on Au, as shown in Fig. 2(b). Apart from oscillations due to the increased Au background, the peak on the high-energy side of the $L_{3}$ edge has vanished, whereas the feature at $778 \mathrm{eV}$ has strongly reduced intensity compared to the multilayer sample. Furthermore, the absence of XMCD
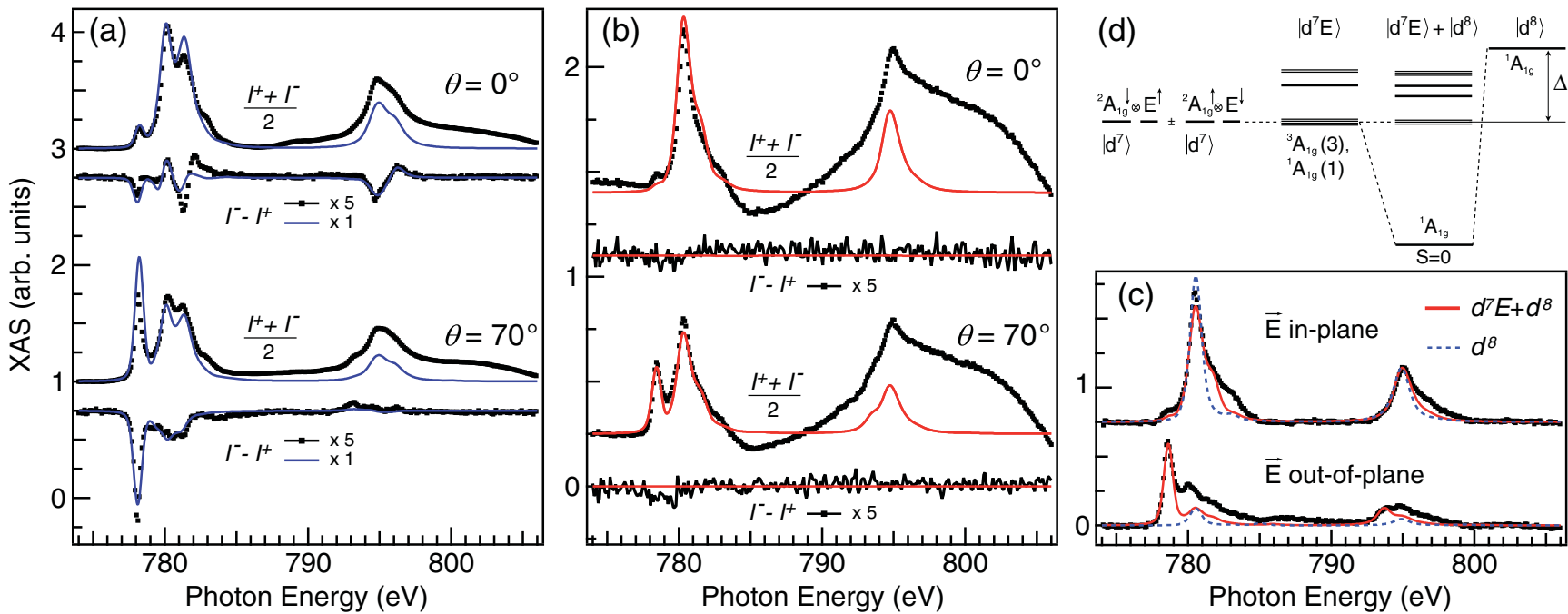

FIG. 2. (Color online) Circularly polarized XAS and XMCD for (a) CoPc multilayer and (b) CoPc monolayer on Au(111). The Co XAS is superposed to a nonlinear background originating from $\mathrm{Au} N$-edge absorption. (c) Linearly polarized XAS of the CoPc monolayer and simulated spectra for $3 d^{8}$ (dashed line) and $3 d^{7} E+d^{8}$ (solid line). (d) Configuration diagram of CoPc/Au showing the coupling of $d^{7}$ and $E$ states (left), pure $d^{8}$ states (right), and $d^{7} E+d^{8}$ mixing (middle). 
intensity both in-plane and out-of-plane indicates that the local magnetic moment of Co is now zero. This result confirms the nonmagnetic behavior of $\mathrm{CoPc} / \mathrm{Au}(111)$ predicted by DFT, but the underlying physics is more involved than simple filling of the $d_{z^{2}}$ orbital described by one-electron models. ${ }^{12,13}$ To obtain insight into the $3 d$ ground state of $\mathrm{CoPc} / \mathrm{Au}(111)$, we attempted first to follow a single configuration approach using either a pure $d^{7}$ configuration, as for the multilayer, or pure $d^{8}$, as expected from DFT. No reasonable agreement could be obtained between experimental and simulated spectra, even using a broad range of crystal-field parameters. $d^{7}$ always produces a finite XMCD intensity due to the odd number of electrons, whereas $d^{8}$ fits neither the intensity and position of the XAS features nor the branching ratio for an $S=0$ configuration. For example, the out-of-plane intensity at $778.5 \mathrm{eV}$ cannot be reproduced using a $d^{8}$ configuration with $S=0$ [dashed line in Fig. 2(c)], since the empty states have in-plane symmetry.

We therefore implemented a CT model accounting for charge fluctuations in the initial and final states. The model assumes a $\mathrm{Co} d^{7}$ configuration with an additional electron in a separate orbital that can hop to the Co ion, thereby creating a $d^{8}$ configuration at a higher energy. The groundstate wave function is a coherent superposition of the form $\alpha\left|d^{7}, 1\right\rangle+\beta\left|d^{8}, 0\right\rangle$, where the additional electron represents the metal states of the surface [Fig. 2(d)]. In the simulations, shown as solid lines in Figs. 2(b) and 2(c), CT was only allowed via the $d_{z^{2}}$ state of the Co ion, since this orbital can overlap with occupied states of the surface $s p$ band. The hopping parameter is $t=1 \mathrm{eV}$ with $\Delta_{i}=0.8$ and $\Delta_{f}=1.3 \mathrm{eV}$ for the effective CT energies between the center of gravity of the initial and final state configurations, respectively. The crystal-field parameters were only little modified from the multilayer values $\left(10 D_{q}=2.6, D_{s}=0.75, D_{t}=0.18 \mathrm{eV}\right)$. We obtain very good agreement with both circularly and linearly polarized XAS, as well as with the XMCD intensity, which is practically zero with residual orbital and spin moments smaller than 0.001 $\mu_{\mathrm{B}}$. The ground state is a spin singlet with $61 \% d^{7}$ and $39 \%$ $d^{8}$ weight. The main effect compared with the multilayer $d^{n}$ configuration is a reduced $a_{1 g}$ hole occupation of $61 \%$ mixed with $d^{n+1}$ states. The quenching of the magnetic moment is very robust as the first excited-state with $S=1$ character lies about $1.1 \mathrm{eV}$ higher in energy. Note that this is not a direct result of the filling of the $d_{z^{2}}$ orbital alone, as the singlet formation occurs also for $d^{8}$ weights of less than $1 \%$, that is, a ground state of almost pure $d^{7}$ character. In other words, coupling to the low-spin $\left|d^{8}, 0\right\rangle$ state forces the singlet formation also of the $\left|d^{7}, 1\right\rangle$ configuration, since mixing and lowering of ground-state energy can only occur if these states have the same symmetry. Such a result may be seen as the electronic analog of a Zhang-Rice singlet, ${ }^{22}$ as strong CoPc-Au hybridization binds an electron to the $\mathrm{Co}^{2+}$ ion to form a local singlet [Figs. 1(b) and 2(d)].

In general, covalency of metal ions in oxides and metalorganic complexes is modeled by configurational mixing with a filled ligand shell as $d^{n}+d^{n+1} L^{-1}$, where $L^{-1}$ denotes a ligand hole. ${ }^{1,15}$ In this way, the total number of electrons of the TM complex is equal to that of the free molecule. For $\mathrm{CoPc}$ on $\mathrm{Au}$, we find that such a scheme does not work, and that an additional electron $E$ has to be considered, giving
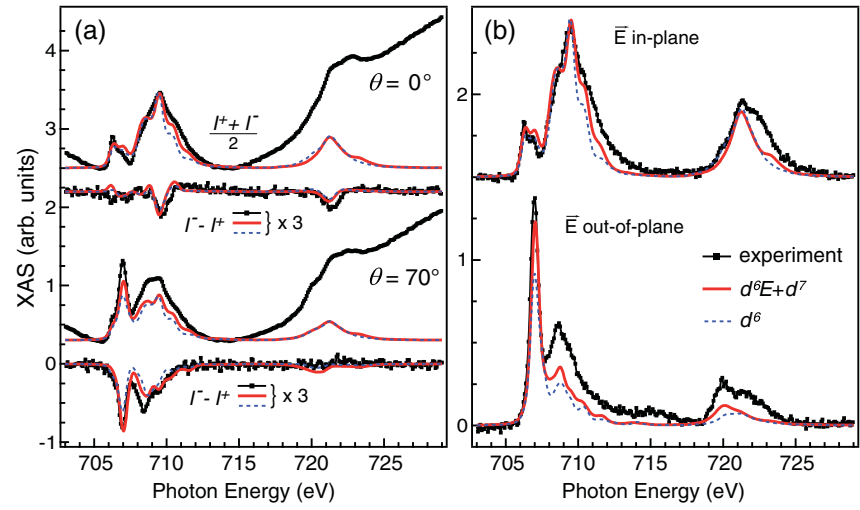

FIG. 3. (Color online) (a) Circularly polarized XAS and XMCD of one monolayer FePc on $\mathrm{Au}(111)$ and (b) linearly polarized XAS. Simulated spectra are shown as dashed $\left(d^{6}\right)$ and solid $\left(d^{6} E+d^{7}\right)$ lines.

$d^{n} E+d^{n+1}$ mixing, as schematized in Fig. 1. Interestingly, the same approach has been employed to model charge fluctuations for TM impurities in metal hosts ${ }^{23,24}$ and bulk ferromagnetic metals, ${ }^{25}$ where an Anderson-like mixing term transfers electrons between the $3 d$ shell and the conduction band, but is treated as a second-order perturbation with respect to leading intraionic interactions. This similarity with metal systems rather than with, for example, oxides, underlines the hybrid character of TM complexes on metal surfaces, for which strong electron correlation and delocalization coexist and determine the overall magnetic behavior.

To test the generality of this model and how the overlap of TM orbitals with surface conduction states influences configuration mixing, we have extended our investigation to a monolayer film of FePc on $\mathrm{Au}(111)$. The XAS line shape, shown in Fig. 3, is remarkably similar to that of thick multilayer films reported in Refs. 26 and 19. Thus, contrary to $\mathrm{CoPc}$, the orbital character of the FePc ground state is not significantly affected by adsorption on $\mathrm{Au}$. Indeed, we find that a simulation based on a single $d^{6}$ configuration with Slater-Condon parameters reduced to $85 \%$ of their atomic value and ground-state crystal field $10 D_{q}=2.7, D_{s}=0.86$, $D_{t}=0.247 \mathrm{eV}$ reproduces the XAS line shape to a good level of accuracy (dashed lines in Fig. 3). ${ }^{27}$ This yields a ground state of mixed ${ }^{3} B_{2 g}{ }^{3} E_{g}$ character with occupation $\left(e_{g}\right)^{2.9}\left(b_{2 g}\right)^{1.3}\left(a_{1 g}\right)^{1.8}$ and $S=1$ intermediate spin. However, the simulations improve when considering CT from the substrate via the $d_{z^{2}}$ orbital, that is, $d^{6} E+d^{7}$ configuration interaction. The CT parameters used in the simulation are $t=1 \mathrm{eV}, \Delta_{i}=3.5$, and $\Delta_{f}=2.5 \mathrm{eV}$, with small changes of $D_{s}=0.85$ and $D_{t}=0.283 \mathrm{eV}$. The $d^{6} E+d^{7}$ spectra (solid lines in Fig. 3) show quantitative agreement of both XMCD and XAS intensity with the experiment and reproduce better the XAS features at the high-energy side of the $L_{3}$ and $L_{2}$ edges. The weight of the $d^{7}$ configuration is small, about $2 \%$, as expected, but charge is transferred from the $e_{g}$ to the $b_{2 g}$ state, leading to $\left(e_{g}\right)^{2.6}\left(b_{2 g}\right)^{1.6}\left(a_{1 g}\right)^{1.8}$ occupation. This has overall little influence on the orbital character of the ground state, but significantly affects the total spin of the FePc/Au complex. The calculations show that the spin of the bound substrate electron couples antiferromagnetically to that of the two lowest energy states of the Fe ion, leading to an effective spin $S=1 / 2$. 
Thus, even a very small mixing can reduce the total magnetic moment of the molecule/surface complex.

Our results present notable differences with respect to recent DFT predictions for $\mathrm{FePc} / \mathrm{Au}(111){ }^{8,13}$ Calculations of the free and adsorbed molecule envisage significant $a_{1 g}$ hole contribution, in contrast with the orbital occupancy found here. Further, DFT predicts a strong change of the Fe electron valence upon adsorption, which is not supported by our measurements. On the other hand, our model provides an interpretation of the magnetic properties of hybrid Pc/metal complexes that is consistent with Kondo physics. The strong mixing between $d^{7} E$ and $d^{8}$ configurations found for $\mathrm{CoPc} / \mathrm{Au}(111)$ indicates that this system belongs to the mixedvalence regime, for which a Kondo resonance is not expected, in agreement with STM experiments. ${ }^{12}$ Conversely, the weak mixing between $d^{6} E$ and $d^{7}$ found for $\mathrm{FePc} / \mathrm{Au}(111)$ shows that this system belongs to the local moment Kondo regime, in accord with local conductance measurements by STM. ${ }^{8}$ The reduced $S=1 / 2$ effective spin of the FePc/Au(111) complex suggests that the Fermi level resonance observed by STM at 5 K (Ref. 8) may be due to partial screening of the $S=1$ spin of FePc, that is, to a manifestation of the underscreened Kondo effect. $^{28}$
In conclusion, we have determined the electronic ground state of $\mathrm{CoPc}$ and $\mathrm{FePc}$ in molecular films and monolayers adsorbed on $\mathrm{Au}(111)$. The nonmagnetic state of $\mathrm{CoPc}$ discussed in previous reports ${ }^{2,12-14}$ is shown to arise from the formation of a local singlet state formed by bound metal and $\mathrm{Co}^{2+}$ electrons. The reduced effective spin of FePc on $\mathrm{Au}$ is compatible with an underscreened Kondo effect. Our results show that charge fluctuations due to electron donation from the surface to TM complexes must be considered within a fully correlated electron scheme in order to reach a satisfactory description of hybrid metal-organic systems. Molecules with symmetry-allowed overlap between empty $3 d$ orbitals and substrate metal states are best described within a mixed valence scheme of type $d^{n} E+d^{n+1}$, where CT from a metallic reservoir acts as a perturbation of many-body ionic orbital levels. The spin multiplicity of the hybrid complexes is determined by that of the higher lying $d^{n+1}$ configuration.

Work was supported by the European Research Council (StG 203239 NOMAD), Ministerio de Ciencia e Innovación (MAT2010-15659), Agència de Gestió d'Ajuts Universitaris i de Recerca (2009 SGR 695), and MIUR (PRIN 20087NX9YT). We acknowledge the ESRF for provision of beam time.
${ }^{1}$ J. Zaanen, G. A. Sawatzky, and J. W. Allen, Phys. Rev. Lett. 55, 418 (1985).

${ }^{2}$ J. Brede, N. Atodiresei, S. Kuck, P. Lazic, V. Caciuc, Y. Morikawa, G. Hoffmann, S. Blugel, and R. Wiesendanger, Phys. Rev. Lett. 105, 047204 (2010).

${ }^{3}$ U. Perera, U. G. E. Perera, H. J. Kulik, V. Iancu, L. G. G. V. DiasdaSilva, S. E. Ulloa, N. Marzari, and S. W. Hla, Phys. Rev. Lett. 105, 106601 (2010).

${ }^{4}$ A. Mugarza, N. Lorente, P. Ordejon, C. Krull, S. Stepanow, M. L. Bocquet, J. Fraxedas, G. Ceballos, and P. Gambardella, Phys. Rev. Lett. 105, 115702 (2010).

${ }^{5}$ K. Moth-Poulsen and T. Bjornholm, Nat. Nanotech. 4, 551 (2009).

${ }^{6}$ V. A. Dediu, L. E. Hueso, I. Bergenti, and C. Taliani, Nat. Mater. 8, 707 (2009).

${ }^{7}$ L. Bogani and W. Wernsdorfer, Nat. Mater. 7, 179 (2008).

${ }^{8}$ L. Gao, W. Ji, Y. B. Hu, Z. H. Cheng, Z. T. Deng, Q. Liu, N. Jiang, X. Lin, W. Guo, S. X. Du, W. A. Hofer, X. C. Xie, and H.-J. Gao, Phys. Rev. Lett. 99, 106402 (2007).

${ }^{9}$ H. Wende, M. Bernien, J. Luo, C. Sorg, N. Ponpandian, J. Kurde, J. Miguel, M. Piantek, X. Xu, Ph. Eckhold, W. Kuch, K. Baberschke, P. M. Panchmatia, B. Sanyal, P. M. Oppeneer, and O. Eriksson, Nat. Mater. 6, 516 (2007).

${ }^{10}$ K. Leung, S. B. Rempe, P. A. Schultz, E. M. Sproviero, V. S. Batista, M. E. Chandross, and C. J. Medforth, J. Am. Chem. Soc. 128, 3659 (2006).

${ }^{11}$ P. A. Reynolds and B. N. Figgis, Inorg. Chem. 30, 2294 (1991).

${ }^{12}$ A. Zhao, Q. Li, L. Chen, H. Xiang, W. Wang, S. Pan, B. Wang, X. Xiao, J. Yang, J. G. Hou, and Q. Zhu, Science 309, 1542 (2005).

${ }^{13}$ Z. Hu, B. Li, A. Zhao, J. Yang, and J. G. Hou, J. Phys. Chem. C 112, 13650 (2008).

${ }^{14}$ B. W. Heinrich, C. Iacovita, T. Brumme, D.-J. Choi, L. Limot, M. V. Rastei, W. A. Hofer, J. Kortus, and J.-P. Bucher, J. Phys. Chem. Lett. 1, 1517 (2010).
${ }^{15}$ F. de Groot and A. Kotani, Core Level Spectroscopy of Solids (CRC Press, Boca Raton, FL, 2008).

${ }^{16}$ See supplemental material at [http://link.aps.org/supplemental/ 10.1103/PhysRevB.83.220401] for information on the STM and low-energy electron diffraction used to monitor the planar growth of the Pc films prior to transfer into the XMCD chamber.

${ }^{17}$ S. Stepanow, A. Mugarza, G. Ceballos, P. Moras, J. C. Cezar, C. Carbone, and P. Gambardella, Phys. Rev. B 82, 014405 (2010).

${ }^{18}$ T. Kroll, V. Yu. Aristov, O. V. Molodtsova, Yu. A. Ossipyan, D. V. Vyalikh, B. Büchner, and M. Knupfer, J. Phys. Chem. A 113, 8917 (2009).

${ }^{19}$ W. Y. P. L. Cook, X. Liu, and F. J. Himpsel, J. Chem. Phys. 131, 194701 (2009).

${ }^{20}$ M.-S. Liao and S. Scheiner, J. Chem. Phys. 114, 9780 (2001).

${ }^{21}$ X. Chen, X. Chen, Y. S. Fu, S. H. Ji, T. Zhang, P. Cheng, X. C. Ma, X. L. Zou, W. H. Duan, J. F. Jia, and Q. K. Xue, Phys. Rev. Lett. 101, 197208 (2008).

${ }^{22}$ F. C. Zhang and T. M. Rice, Phys. Rev. B 37, 3759 (1988).

${ }^{23}$ L. L. Hirst, Adv. Phys. 27, 231 (1978).

${ }^{24}$ D. C. Abbas, T. J. Aton, and C. P. Slichter, Phys. Rev. Lett. 41, 719 (1978).

${ }^{25}$ T. Jo and G. A. Sawatzky, Phys. Rev. B 43, 8771 (1991).

${ }^{26}$ J. Bartolomé, F. Bartolome, L. M. Garcia, G. Filoti, T. Gredig, C. N. Colesniuc, I. K. Schuller, and J. C. Cezar, Phys. Rev. B 81, 195405 (2010).

${ }^{27}$ For FePc, we used final-state crystal-field parameters reduced by $10 \%-20 \%$ with respect to the initial state to account for the contraction of the $d$ states induced by the core hole. This effect is not relevant for $\mathrm{CoPc}$, as the lower energy levels are not as close to each other.

${ }^{28}$ P. Nozières and A. Blandin, J. Physique 41, 193 (1980). 